\title{
Hydrodynamic Properties of Benthic Marine Crustacea. I. Specific Gravity and Drag Coefficients
}

\author{
D. H. Spaargaren \\ Netherlands Institute for Sea Research, Postbox 59, Texel, The Netherlands
}

\begin{abstract}
The specific gravity of various species of marine, benthic crustaceans is determined in relation to body size. The data obtained are compared with the mode of life (swimming capabilities; distribution) and osmoregulatory capacities (hypotonicity) of the species examined. Within a species, density appears to be independent of size or sex; possible interspecific differences are masked by large intraspecific variation. Interspecifically, body density appears to be related to the drag coefficient for sinking, whereas the expected relation between density and hypotonicity is lacking. The relation between density and drag coefficient is supported by the finding that sinking rates of various animals are practically independent of their densities; species with relatively high densities are not necessarily confined to bottom dwelling modes of life. Drag coefficients appear to be considerably larger than those predicted by Stokes' law; depending on the species, the difference may amount to a factor of 60 to 350 . The influence of the body weight recorded suggests a dependence on body surface area. In interspecific comparison, species-specific drag coefficients (drag coefficients corrected for medium viscosity and animal weight) do not show the expected negative relation with sinking rate. In part, this can be explained by the interspecific, positive relation between drag coefficients and densities: a more complicated body shape is related to larger dimensions of the (heavy) exoskeleton, resulting in higher densities.
\end{abstract}

\section{INTRODUCTION}

The body density of an aquatic organism may play an important role in its distribution. When, like in many benthic crustaceans, parts of the active periods are spent by swimming around freely, a low density not much deviating from that of the surrounding water - will be an advantage in remaining in suspension. In this connection a heavy exoskeleton would be a disadvantage.

On the coast or in estuaries, in some shrimp species, such as the common shrimp Crangon crangon, individuals of similar sizes are often found together: larger individuals in deeper water, smaller ones in shallower water. With exceptions, the same phenomenon can also be observed in other marine species. Various explanations exist for these peculiar size distributions, but they are not quite satisfactory. Most simply one might think that such size-related selection is maintained passively, due to body-density differences. On the one hand it is possible that, with increasing body size, due to a decreasing surface/volume ratio, the influence of the exoskeleton on the density becomes smaller; on the other hand, especially in larger ani- mals, a higher rigidity of the exoskeleton may be required, thus increasing the contribution of the exoskeleton to overall body density. In animals with poor swimming capabilities, mainly transported passively by water currents, differences in density between smaller and larger individuals may contribute to differences in their distribution.

Generally, data on intraspecific and interspecific density variation in marine Crustacea are scarce. Some density values for marine invertebrates were presented by Lowndes $(1938,1942,1943)$. In several papers Denton and Gilpin-Brown deal with problems of density regulation in cephalopods (and fishes) (e.g. Denton, 1961; Denton and Gilpin-Brown, 1961). Childress and Nygaard (1974) determined the specific gravity and chemical composition of 27 species of bathypelagic crustaceans. Benthic Crustacea received little attention in this respect.

Previous work showed that the density of marine invertebrates is usually higher than that of sea water; even pelagic species tend to sink (this contrasts to the situation prevailing in most fishes which, by means of gases in their swim bladder, can regulate their body density to a value close to that of sea water). In species 
with a high water content (e.g. coelenterata), body density and medium density are nearly the same. The presence of a gas-filled skeleton (e.g. in Sepia) or a high content of light cations (e.g. $\mathrm{NH}_{4}^{+}$), urea or a low content of heavy cations (e.g. $\mathrm{Ca}^{++}$; see Gross and Zeuthen, 1948) or an overall low electrolyte content of the body fluids may have a similar effect. A high fat content, also decreasing body density to values more closely resembling that of sea water, seems, at least in Crustacea, of less importance (Culkin and Morris, 1969): In 7 crustacean species from the East-Atlantic Ocean, only small amounts of phospholipids, monoglycerides and sterols were found (between 2 and $5 \%$ of the total body weight). However, differences in the contribution of mono-saturated fatty acids to total fat content are found in some copepod species inhabiting various depths (Morris, 1971). Gross and Raymond (1942) described a decrease in density of Stage V Calanus finmarchicus in winter; this corresponds to an increase in fat content.

In this study, additional data on the density of some benthic crustaceans are presented in relation to body size, in order to (1) find out whether changes in density occur during ontogenetic development, and (2) compare the values for the different species in relation to their mode of life and distribution.

When an aquatic animal, after a period of swimming, stops its locomotory movements, it will usually attain, almost instantaneously, a constant sinking rate. Especially in semi-benthic shrimps such movement pattern is part of the normal locomotory behaviour. Sinking proceeds very smoothly, without turbulent oscillations, while the geometric position of the body axis does not alter.

It is clear that sinking rate, in one way or another, is related to the ease with which an animal can swim and maintain a fixed vertical position in the water column. An animal which, in the absence of swimming movements, sinks only very slowly, or does not sink at all, will have to spend little or no energy to remain suspended. It is difficult to determine the exact relation between sinking rate and energy requirements for swimming (e.g. Denton, 1961; Wu, 1977). The conversion efficiency of metabolic energy to locomotory energy is difficult to assess. Studies of these problems are mainly confined to teleosts (e.g. several papers in Pedley, 1977). Attempts to predict the minimal power requirements were made by Spaargaren (1980).

The velocity with which an animal sinks passively is partly determined by its underwater weight. It also depends on the frictional resistance between animal and surrounding medium. The latter is given by the drag coefficient for sinking which (in non-turbulent conditions) indicates the ratio between friction force $F_{f}$ (dyne), and velocity $v\left(\mathrm{~cm} \mathrm{sec}{ }^{-1}\right)$, with regard to the medium. For spherical abjects in laminar flow the drag coefficient $k$ can be deduced from Stokes' law: $k=$ $6 \pi r \eta$ ( $r$, radius of the sphere; $\eta$ : medium viscosity). Empirically, the drag coefficient can be easily obtained from underwater weight and sinking rate; at a constant sinking rate the frictional force equals the underwater weight $\left(k=F_{f} / v\right)$.

Various examples provided in the literature describe relations between body shape, friction forces and swimming abilities of aquatic animals. Well known are changes in body shape of certain plankton organisms related to seasonal changes in temperature (density) of the medium. Quantitative data on drag coefficients, however, are scarce. Chamberlain (1976) and Scott and Chamberlain (1976) give values for cephalopods. The present paper also gives additional data on the drag coefficients for passive sinking in a number of marine and estuarine Crustacea.

\section{MATERIAL AND METHODS}

\section{Material}

Data on hydrodynamic properties were obtained for the following species: Palaemon serratus (Pennant), Lysmata seticaudata (Risso), Sicyonia carinata (Olivi), Pontophilus spinosus (Leach), and Maia squinado (Herbst). These crustaceans were collected in the autumn of 1977 in the bay of Naples (Italy), where they are common. In spring 1978, Crangon crangon (L.) was collected in the North Sea, near the island Texel; and in summer 1978, Praunus flexusos (Müller), Idothea balthica (Pallas), Homarus vulgaris (L.), and Palaemon adspersus (L.) were obtained from the Øresont, near Helsingør (Denmark).

Density as well as drag coefficients were determined in water in which the animals mentioned naturally occur. When possible, test animals of widely diverging sizes were selected.

\section{Density Determinations}

As the differences in density could be expected to be small, an accurate method for density determination was of utmost importance. In principle, several methods might be used; most involve a separate determination of volume and weight. In the weight measurement of an aquatic animal there is the problem of adhering water which cannot be wiped off. Especially in Crustacea with inaccesible gill chambers this may strongly influence the accuracy. The method described here avoids this problem to a large extent: Individuals were weighed in a small vessel (Fig. 1), 


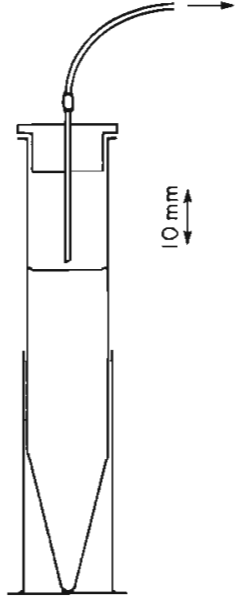

after removal of most of the adhering water with absorbing paper. Subsequently, the volume of the animal plus the remaining adhering water was determined. Sea water was added to the vessel up to about $1 \mathrm{~cm}$ below the rim; then, by placing a lid on top of the vessel, the volume is reduced to a very accurately fixed value by sucking water through the capillary mounted in the lid until the water level is lowered to such an extent that air begins to enter the capillary. The weight

Fig. 1. Vessel used for density determination of shrimps and prawns (for description see text)

increase, by the addition of sea water up to the fixed volume, is substracted from that determined in the same way in the absence of an animal. The volume is obtained by dividing the difference by the density of the sea water used. Weight and volume, measured immediately after one another, include the adhering water. The relative error $(R E)$ introduced by the presence of an unknown, but equal amount of adhering water, in the final value for the density $\left(S_{a}\right)$ will be:

$$
R E=\frac{x\left(1-\frac{S_{m}}{S_{a}}\right)}{1+x} \cdot 100 \%
$$

$\left(S_{m}, S_{a}\right.$ represent, respectively, the density of the medium and the animal; the equation assumes the amount of adhering water to be a certain fraction, $x$, of the volume of the animal.) The differences between the densities of medium and animals are small, seldom exceeding $15 \%$. If, for instance, one assumes $10 \%$ adhering water in an animal having a density of 1.1318 $\mathrm{g} \mathrm{ml}^{-1}$ (average value for Palaemon serratus - see results - which shows a relatively large difference from the density of its medium) then $R E=0.86 \%$ follows. This shows that, even under very unfavourable conditions, the density can be determined with an accuracy of better than $1 \%$ if adhering water is included in both, weight and volume measurements. This is not the case when weight is determined in air and volume is deduced from the weight decrease at submersion (e. $\mathrm{g}$. Childress and Nygaard, 1974).

\section{Drag Coefficient Determinations}

Sinking rates of (anaesthesized) animals were determined by using a tube, diameter $10 \mathrm{~cm}$, length $150 \mathrm{~cm}$.
In the wall of the tube paired, metal (platinum) electrodes were mounted, between which the electrical capacity was measured by means of a universal measuring bridge (Wayne Kerr, Type B 642). Changes in capacity at the passage of an animal were recorded (Kipp and Zn, recorder Type BD9). Paper transport was switched on when the animal started to sink in the tube. When introduced into the tube, the animals always assume practically instantaneously their normal geometric position, the length-axis horizontal and remain stable during sinking. In some instances, animals turned on their back, but, this was found not to affect their terminal velocity; the length-axis remained horizontal in all cases. The quotient of underwater weight and sinking rate thus obtained yields the drag coefficient for laminar flow.

\section{RESULTS AND DISCUSSION}

\section{Density}

The results of the density determinations in 4 species are, as a function of body size, given in Figure 2. It is clear that there is no significant influence of body size on animal density or that this is masked by large individual variation (generally the regression lines show a negative slope, however, the correlation was never found to be significant). This means that during the ontogenetic development of the animals concerned no changes in chemical composition occur which are strong enough to influence their densities. Table 1 shows average density values with their standard deviations, obtained for various species including animals of different sizes.

The intraspecific variation in density is smallest in Lysmata seticaudata where the variation probably only reflects measuring accuracy. Other species show a much larger variation; it is not clear to which cause (feeding condition, stage in the moult cycle, etc.) this larger variation must be ascribed. The intraspecific variation is so large that interspecific differences between the average values of the various species considered here are only slightly significant or not significant at all.

The density of the body constituents is generally higher than that of sea water (the latter varies between 1.023 and $1.031 \mathrm{~g} \mathrm{ml}^{-1}$ in $34.4 \%$ S at $25^{\circ} \mathrm{C}$ and $38.9 \%$ $\mathrm{S}$ at $0{ }^{\circ} \mathrm{C}$, respectively). An important contribution to the density of an animal is made by its body fluids, which often comprise more than $65 \%$ of the body weight. One might expect a difference between the strongly hyporegulating Palaemon serratus and other, osmoconforming species like Lysmata seticaudata, Sicyonia carinata and Maia squinado. The water con- 


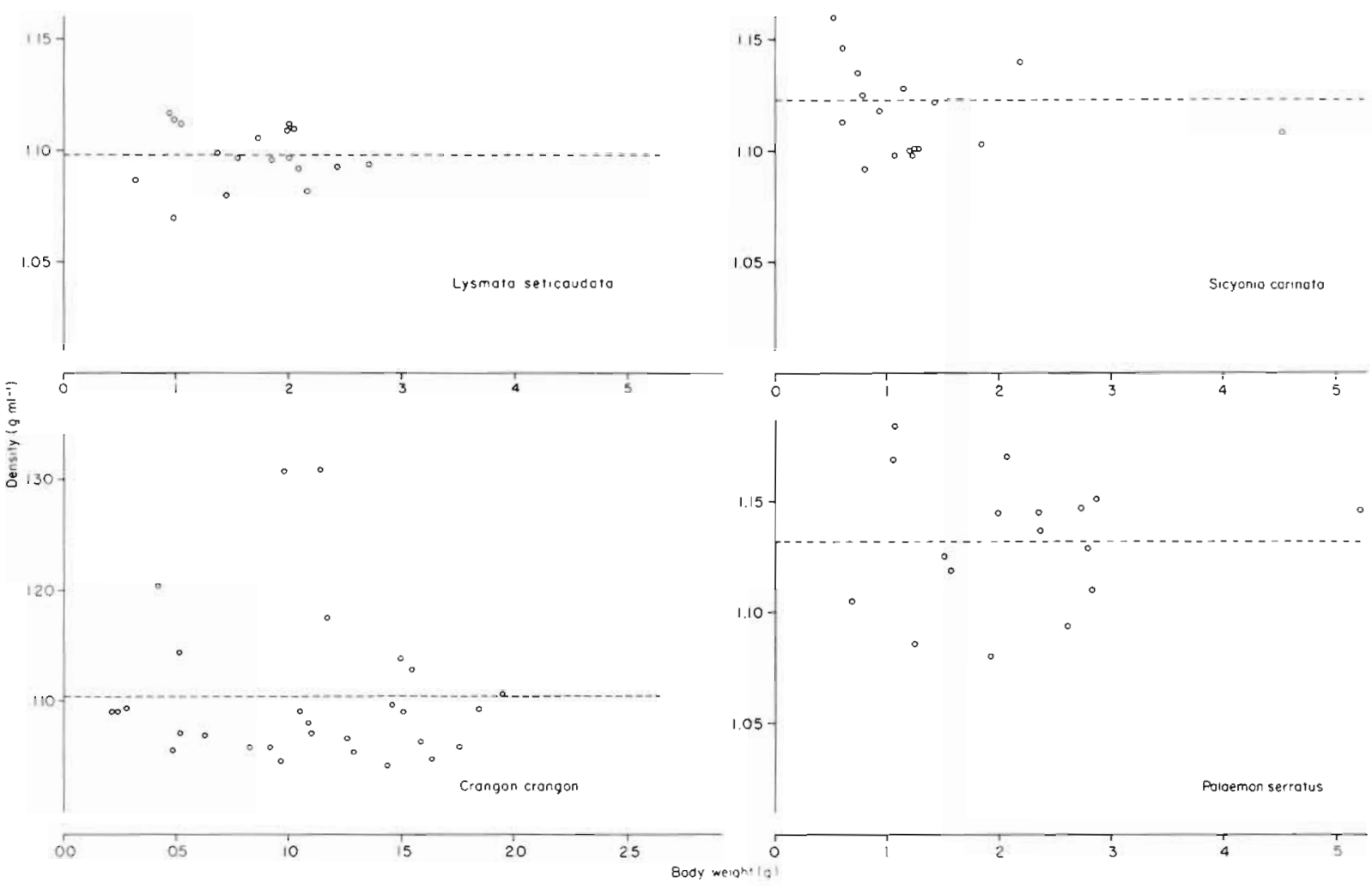

Fig. 2. Relation between density and body weight in 4 crustaceans

tent of these species is approximately the same, ca $70 \%$ (Spaargaren, 1972). It appears, however, that the density of $P$. serratus is not exceptionally low. Compared to the osmoconforming species, there is no significant difference (the average value is even higher). Evidently the effect of a low electrolyte concentration in the blood of $P$. serratus is masked by the presence of other heavy constituents.

The density of the various species is remarkably similar. In the larger species (crabs, lobsters) density is slightly higher than in the smaller species (shrimps, prawns); the effect of a heavier exoskeleton in animals of larger size is hardly reflected in their density. However, in comparing the extreme density values it appears that the lowest values occur in the small, pelagic species, whereas the highest density values are found in the larger benthic species belonging to the genera Maia, Carcinus, Cancer; this indicates a

Table 1. Density of crustaceans $\left(20^{\circ} \mathrm{C}\right) . s$ : standard deviation; $N$ : number of measurements

\begin{tabular}{|c|c|c|c|c|}
\hline Species & $\begin{array}{c}\text { Density } \\
\mathrm{g} \mathrm{ml}^{-1}\end{array}$ & $s$ & $N$ & Reference \\
\hline Calanus finmarchicus & 1.506 & & & Gross and Raymond, 1942 \\
\hline Lysmata seticaudata & 1.098 & 0.013 & 18 & This paper \\
\hline Hemimysis lamornae & 1.104 & & & Lowndes, 1942 \\
\hline Crangon crangon & 1.104 & 0.068 & 29 & This paper \\
\hline Pontophilus spinosus & 1.1148 & & 1 & This paper \\
\hline Homanus vulgaris & 1.1170 & & & Cited in Nicol, 1960 \\
\hline Sicyonia carinata & 1.1226 & 0.031 & 19 & This paper \\
\hline Palaemon serratus & 1.1318 & 0.030 & 17 & This paper \\
\hline Praunus flexuosus & 1.1474 & 0.030 & 4 & This paper \\
\hline Idothea balthica & 1.1460 & 0.049 & 5 & This paper \\
\hline Maia squinado & 1.1812 & 0.151 & 4 & This paper \\
\hline Carcinus maenas & 1.2076 & & & Cited in Nicol, 1960 \\
\hline Palaemon adspersus & 1.2644 & 0.073 & 5 & This paper \\
\hline Cancer pagurus & 1.2784 & & & Cited in Nicol, 1960 \\
\hline Sea water $(35 \%$ S $)$ & 1.0243 & & & Riley and Skirrow, 1975 \\
\hline
\end{tabular}




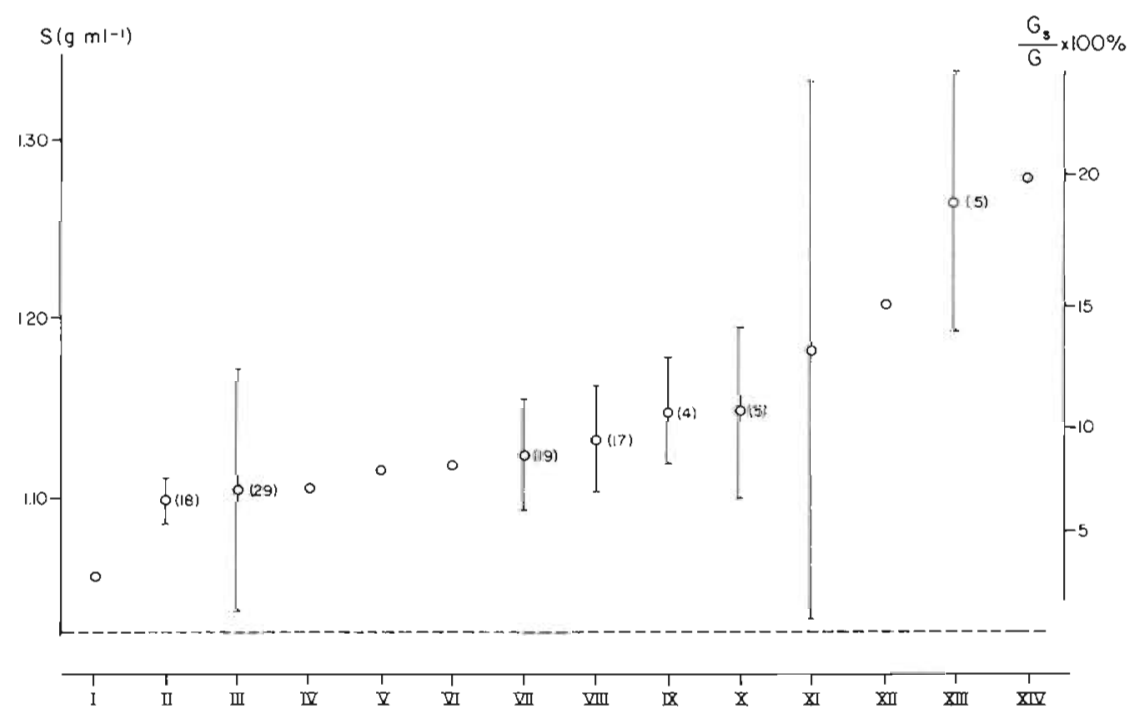

Fig. 3. Interspecific comparison of body density of 14 crustaceans. Vertical bars: standard deviation; bracketed numbers: number of measurements. I: Calanus finmarchicus; II: Lysmata seticaudata; III: Crangon crangon; IV: Hemimysis lamornae; V: Pontophilus spinosus; VI: Homarus vulgaris; VII: Sicyonia carinata; VIII: Palaemon serratus; IX: Praunus flexuosus; X: Idothea balthica; XI: Maia squinado; XII: Carcinus maenas; XIII: Palaemon adspersus; XIV: Cancer pagurus

oXI
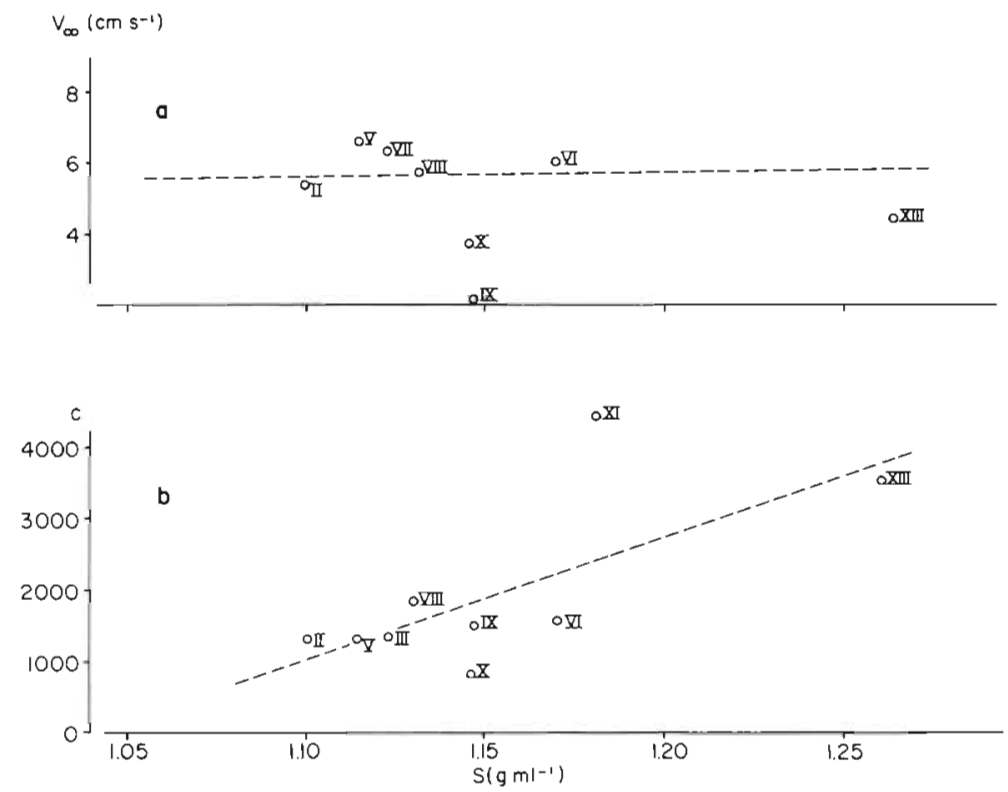

Fig. 4. Sinking velocity $\left(v_{c o}\right)$ in the absence of swimming movements (a), and drag coefficients (b) of crustaceans (fresh weight $1 \mathrm{~g}$ ) in relation to body density. Roman figures: species as indicated in legend to Figure 3

lose relation between density and swimming capabilities.

Very remarkable is the interspecific relation between density and drag coefficient for passive sinking. The latter determines the velocity an animal attains when, after swimming, it stops its locomotory activity and sinks towards the bottom. In species with a relatively high density, the drag coefficient ( $c$, drag coefficient corrected for medium viscosity and animal weight; see below, Table 3) is also high (Fig. 4b); hence, sinking rate becomes more or less independent of the animal's density (Fig. 4a). Lowndes (1942) introduced the expression 'sinking factor', being the ratio between the density of the animal and that of the medium (times 1000). From the relation mentioned above it will be clear that sinking rate may not be related to the sinking factor.

In conclusion, in the crustaceans considered the significance of body density is limited. Differences in body composition affect density only to a small extent. 
Furthermore, effects of density differences on underwater weight and sinking rate can be compensated by other hydrodynamic properties (body shape), reflected in the drag coefficient. This makes the ultimate role that density plays in determining the swimming capabilities and the distribution of a species very complex. In fact the significance of density can only be expressed in relation to other properties of the animal concerned.

\section{Drag Coefficients}

As mentioned above, the drag coefficient is a function of the ratio underwater weight to sinking rate. This statement assumes a non-turbulent movement during which the frictional resistance force $\left(F_{t}\right)$ is linearly related to the sinking rate, $v: F_{f}=\mathrm{k} \cdot \mathrm{v}$. In this case, sinking rate is given by:

$$
v_{t}=m g k^{-1}\left(1-S_{m} / S_{a}\right)(1-\exp k t / m)
$$

After some time the sinking rate becomes constant:

$$
v_{\infty}=m g k^{-1}\left(1-S_{m} / S_{a}\right)
$$

the half-time for reaching equilibrium follows from $t_{1 / 2}$ $=0.693 \mathrm{~m} / \mathrm{k}(\mathrm{m}$ : mass; $g$ : gravity constant $; \mathrm{k}$ : drag coefficient; $t$ : time; $S_{m}$ and $S_{a}$ : density of medium and animal, respectively).

It is questionable whether the assumption of nonturbulent flow is justified considering the animals used here. Turbulent movement implies that the friction force is proportional to the square of the velocity: $F_{f}=$ $k^{\prime} \cdot v^{2}$. In that case velocity is given by:

$$
v_{t}=v_{\infty} \cdot \tanh \left|\frac{k^{\prime} g}{m}\left(1-\frac{S_{m}}{S_{a}}\right)\right| 0.5 \cdot t
$$

and terminal velocity follows from:

$$
V_{\infty}=\sqrt{\frac{m g}{k^{\prime}}\left(1-\frac{S_{m}}{S_{a}}\right)}
$$

Half-time for reaching equilibrium is shorter and can be deduced from:

$$
t_{1 / 2}=0.549\left|\frac{\mathrm{k}^{\prime} \mathrm{g}}{\mathrm{m}}\left(1-\frac{S_{\mathrm{m}}}{S_{a}}\right)\right|^{-0.5}
$$

In order to find out which drag coefficient, $k$ or $k^{\prime}$, should be determined, the underwater weight of an animal was varied by inserting small weights into its gill chambers (Fig. 5). At terminal velocity, underwater weight equals the frictional resistance force. It appears that the resistance force is linearly related to the velocity attained: $F_{f}=k \cdot v$, even at high final velocities. Therefore, there is no reason to assume that in the species used (Crangon crangon) turbulent flow occurs, It was assumed that the same holds for the other species considered. This seems justified since prob-

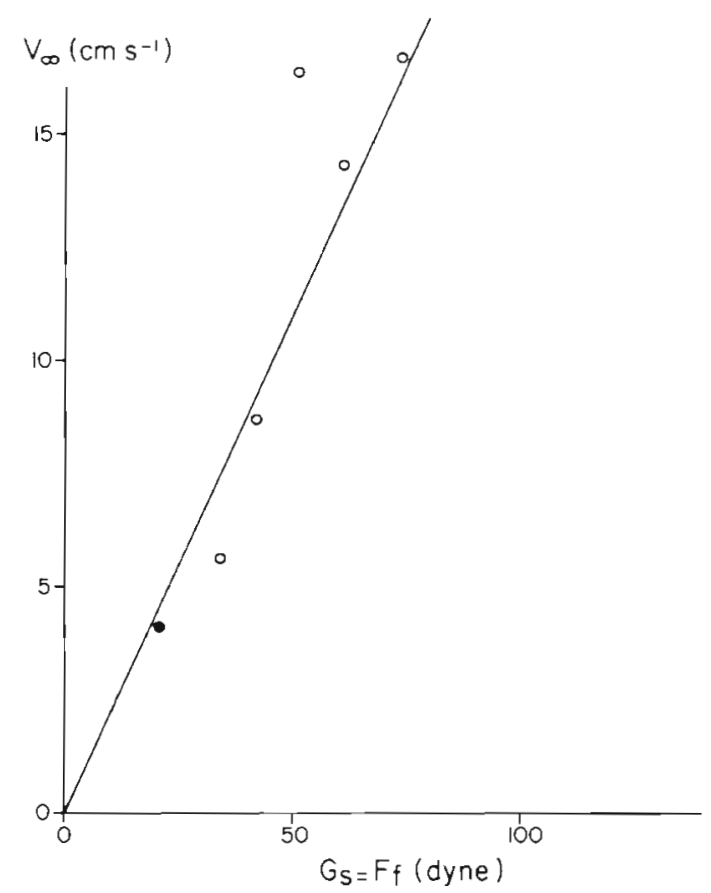

Fig. 5. Crangon crangon. Terminal sinking rate $\left(v_{\infty}\right)$ in relation to underwater weight $\left(G_{s}\right)$. Individual with fresh weight of $359 \mathrm{mg}$ and small weights inserted (o) into its branchial chambers. At terminal velocity underwater weight equals frictional resistance force $\left(F_{t}\right)$ of water passing the animal.

- Individual without additional weight. $20^{\circ} \mathrm{C} ; 32.3 \% \mathrm{~S}$

ably in all cases the Reynolds number $\left(R e=x \cdot V \cdot s_{m} / \eta\right.$; $\mathrm{x}$, size; $\eta$, viscosity) remains low, not exceeding a value of about $1000 ; 2000$ is regarded the limit between nonturbulent and turbulent flow (e.g. Glastone and Lewis, 1966; however, often also lower values - Re $<1$ - are frequently used). Table 2 lists $k$ and $k^{\prime}$ values obtained for the various species. Below only the drag coefficient for nonturbulent flow, $k$ will be used.

With the animals considered here terminal sinking rate is reached very soon, long before the upper electrodes of the tube are passed. This becomes evident from the equal time intervals measured between the passages of the electrode pairs mounted at equal distances in the lower part of the tube. Sinking rate values, measured for different species of various sizes, ranged between 2 and $12 \mathrm{~cm} \mathrm{~s}^{-1}$.

Figure 6 shows examples of sinking rates measured in 4 shrimp species using individuals of different body weights. From this it is clear that the increase in sinking rate at increasing (underwater) weight is not the same in different species: an increase in underwater weight results in higher sinking rates in Palaemon serratus and Sicyonia carinata, but in the others sinking rate appears to remain constant over a wide range of underwater weights. In the latter species the increase in weight seems to be compensated for by a 


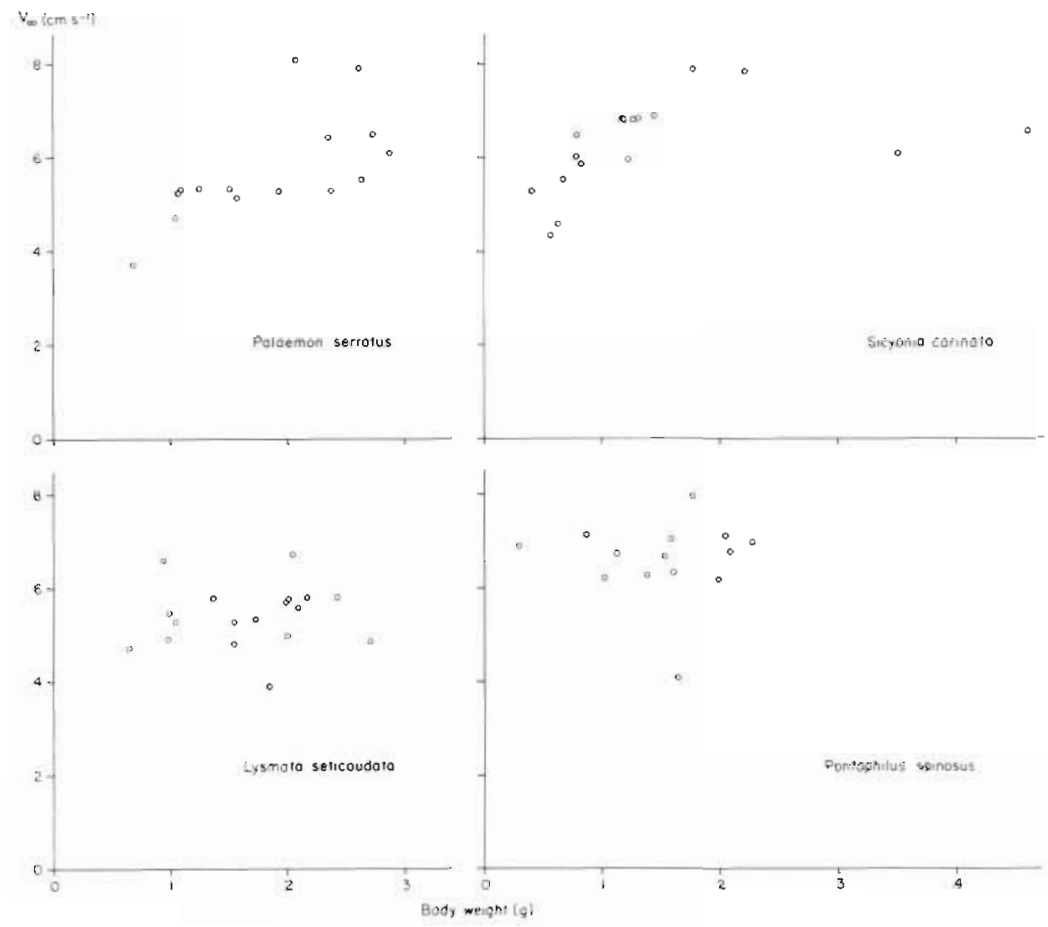

Fig. 6. Examples of terminal sinking rates as function of body weight in 4 crustaceans. $20^{\circ} \mathrm{C} ; 38.4 \%$

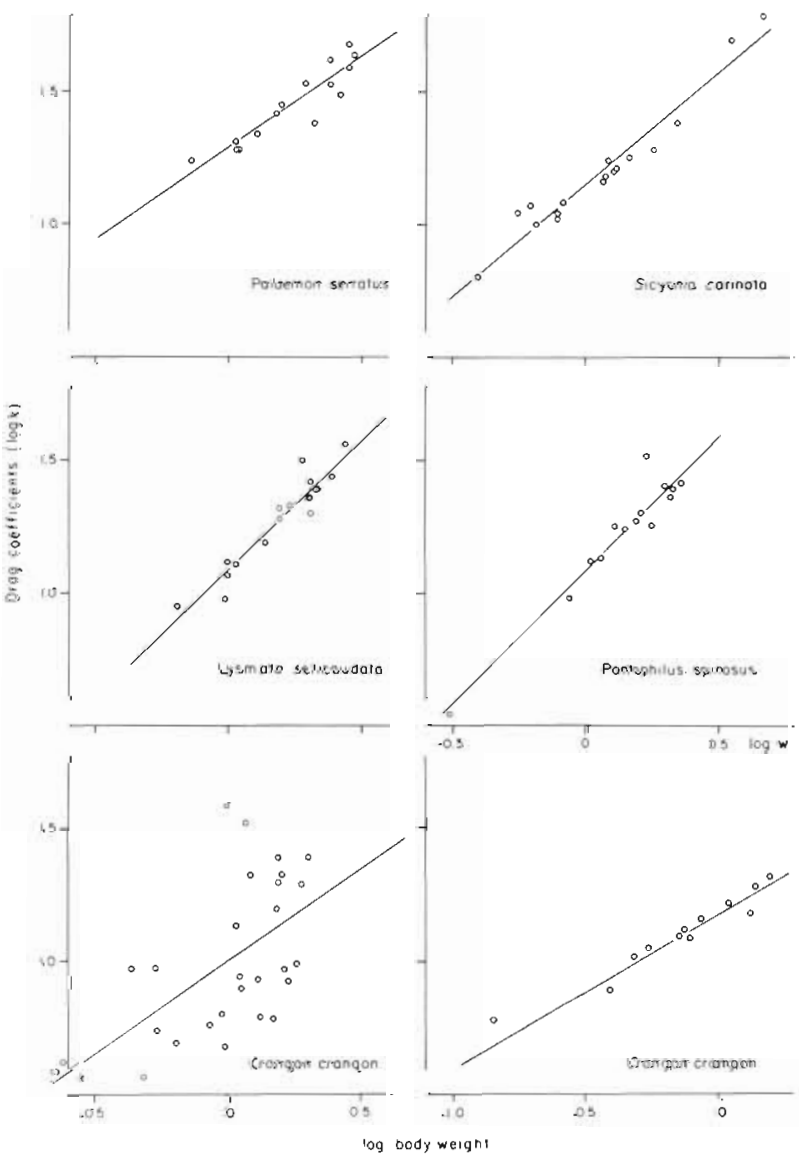

Fig. 7. Drag coefficients as a function of body weight in 5 crustaceans. $20^{\circ} \mathrm{C}$ (except Crangon crangon bottom right: 5 ${ }^{\circ} \mathrm{C}$ ); $38.4 \% \mathrm{~S}$ (except both C. crangon subfigures: $30 \% \mathrm{~S}$ ) higher frictional resistance, as it appeared above that density of these species is not related to body size.

According to Figure 6 the ratio between sinking rate and underwater weight may be non-linearly related to the body weight (size). The relation between drag coefficient and body weight could, therefore, better be represented by: $k=a w^{b}$, where $a$ and $b$ are constants. Figure 7 shows some examples in which $\log k$ is plotted against $\log w$ (slope and Y-intercept yield values for $a$ and $b$, as indicated in Table 2). Although it would be expected that the animals normally vary their frictional resistance by changing the geometric position of their appendages, it can be seen that the variation in $k$, found for (anaesthesized) animals of similar size, is relatively low compared to the variation related to size differences.

The values obtained for the drag coefficients strongly deviate from those calculated according to Stokes' law. Firstly, the influence of weight is considerably larger: for the exponent, $b$, values between 0.6 and 1.0 are found, while Stokes' law predicts a relation with weight to the power $1 / 3(\mathrm{k}=6 \pi r \eta=6 \pi \eta$ $\left(S_{a} \cdot \frac{4}{3} \cdot \pi\right)^{-1 / 3} \cdot w^{1 / 3} ;$ for example, with animal density $S_{a}=1.13$ and viscosity $\eta=1.06$ centipoise follows $k=0.12 w^{1 / 3}$ ). Values found for the coefficient a are regarding an animal of $1 \mathrm{~g}$ weight -60 to 350 times larger than those predicted by Stokes' law.

The drag coefficients $k$ are proportional to medium viscosity. This is evident from values determined in Crangon crangon at low and high temperature: at $5{ }^{\circ} \mathrm{C}$ $k=15.28 w^{0.597}$ and at $20{ }^{\circ} \mathrm{C} k=10.17 w^{0.699}$. If the $k$ - 
Table 2. Drag coefficients $k\left(k=a w^{b}\right)$ and $k^{\prime}\left(k=a^{\prime} w^{b}\right)$ as a function of animal weight ( $w$, in $\left.g\right)$ in the crustaceans listed. Assuming laminar flow $(k)$ and turbulent flow $\left(k^{\prime}\right)$ drag coefficients were derived by correlating terminal sinking rate with animal weight in the equations $\log w_{s} g / v w=(b-1) \log w+\log a$ and $\log w_{s} g / v^{2} w=(b-1) \log w+\log a$ ( $w_{s}$ represents underwater weight). In all cases correlation coefficient ( $r)$ remained the same whether assuming laminar or turbulent flow. N: number of observations

\begin{tabular}{|c|c|c|c|c|c|c|c|c|}
\hline Species & $\begin{array}{c}\text { Temp. } \\
\left({ }^{\circ} \mathrm{C}\right)\end{array}$ & $\begin{array}{l}\text { Sal. } \\
(\% \infty S)\end{array}$ & $a$ & $b$ & $a^{\prime}$ & $b$ & $r$ & $N$ \\
\hline Maia squinado & 20 & 38 & 8.62 & 1.087 & 0.50 & 1.175 & 0.2026 & 4 \\
\hline Crangon crangon & 20 & 30 & 10.17 & 0.699 & 1.40 & 0.398 & 0.5606 & 29 \\
\hline Idothea baIthica & 20 & 30 & 10.60 & 0.747 & 1.06 & 0.496 & 0.8113 & 10 \\
\hline Pontophilus spinosus & 20 & 38 & 11.89 & 1.000 & 1.83 & 1.000 & 0.0004 & 13 \\
\hline Lysmata seticaudata & 20 & 38 & 12.03 & 0.968 & 2.28 & 0.937 & 0.0972 & 18 \\
\hline Sicyonia carinata & 20 & 38 & 13.80 & 0.842 & 2.28 & 0.683 & 0.6224 & 17 \\
\hline Crangon crangon & 5 & 30 & 15.28 & 0.597 & 3.15 & 0.194 & 0.9526 & 12 \\
\hline Homarus vulgaris & 20 & 30 & 16.54 & 0.655 & 2.17 & 0.311 & 0.9682 & 4 \\
\hline Palaemon serratus & 20 & 38 & 19.13 & 0.685 & 4.03 & 0.370 & 0.7278 & 15 \\
\hline Praunus flexuosus & 20 & 30 & 31.66 & 0.862 & 9.20 & 0.724 & 0.4916 & 8 \\
\hline Palaemon adspersus & 20 & 30 & 43.18 & 0.738 & 9.81 & 0.484 & 0.7980 & 9 \\
\hline
\end{tabular}

values obtained are divided by the medium viscosity we find: $k_{5} / \eta_{5}=986 w^{0.597}$ and $k_{20} / \eta_{20}=959 w^{0.699}$. The differences between these drag coefficients, corrected for medium viscosity, are ascribed to measurement inaccuracies.

The values obtained for the exponent $b$ suggest a relation with the surface area of the animal (proportional to $w^{2 / 3}$ ). Table 3 gives the drag coefficient $c$ corrected for medium viscosity, assuming that $k$ is related to $w^{2 / 3}: c=k / \eta \cdot w^{2 / 3}$.

Table 3. Shape constants (c) of the crustaceans listed; drag coefficient $\mathrm{k}=\mathrm{c} \cdot \eta \cdot \mathrm{w}^{2 / 3} \cdot \mathrm{s}$; standard deviation; $N$ : number of observations

\begin{tabular}{|lrrr|}
\hline \multicolumn{1}{c}{ Species } & \multicolumn{1}{c}{$c$} & \multicolumn{1}{c}{$s$} & $N$ \\
\hline Idothea balthica & 725 & 89 & 10 \\
Crangon crangon & 1256 & 225 & 29 \\
Lysmata seticaudata & 1291 & 231 & 18 \\
Pontophilus spinosus & 1294 & 268 & 13 \\
Sicyoni carinata & 1329 & 255 & 17 \\
Praunus flexuosus & 1479 & 118 & 8 \\
Homarus vulgaris & 1559 & 135 & 3 \\
Palaemon serratus & 1803 & 231 & 15 \\
Palaemon adspersus & 3495 & 1373 & 9 \\
Maia squinado & 4405 & 1052 & 4 \\
& & & \\
\hline
\end{tabular}

The value for the shape constant $c\left(c \cdot w^{2 / 3}\right.$ compar. able with $6 \pi$ in Stokes' law for spherical objects) only depends on the shape of the animal. Interspecific comparison reveals: (1) The shape constant $c$ is not strongly related to the sinking rate of an animal (Fig. 8); in contrast to the expected negative relation there is a slight tendency indicating that animals with a higher shape constant have a higher sinking rate. (2) The shape constant appears to be positively related to body

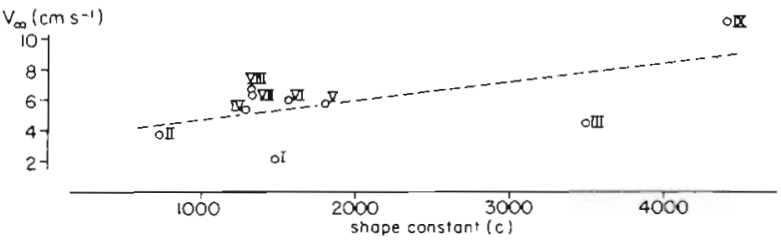

Fig. 8. Relation between sinking rate and shape constant (c) in crustaceans. I: Praunus flexuosus; II: Idothea balthica; III: Palaemon adspersus; IV: Lysmata seticaudata; V: Palaemon serratus; VI: Homarus vulgaris; VII: Sicyonia carinata; VII: Pontophilus spinosus; IX: Maia squinado. Linear correlation coefficient, $r=0.6955 ; N=9$

density (Fig. 4b); this can be explained by the exoskeleton affecting density. (3) The shape constant appears to be positively related to average body size (linear regression coefficient $0.7760, N=9$ ); this means that, irrespective of the weight influence on the drag coefficient the larger species considered here appear to have higher drag coefficients.

As mentioned in the introduction the drag coefficients as derived above have significance for the swimming abilities of an animal. Probably a simple relation exists between the (minimal) energy requirement to maintain a vertical position in the water column $(P)$ and the inverse of the drag coefficients $\left(k=a w^{b}\right.$ or $k=$ $c \eta w^{2 / 3}$ ) as indicated above: $P \approx$ (underwater weight) ${ }^{2}$; drag coefficient. This aspect will be subject of further study.

Acknowledgements. I am much indebted to Dr. L. Hagerman (Marine Biological Laboratory, Helsingor, Denmark) as well as to staff and personnel of the Stazione Zoologica, Naples (Italy) for help in collecting animals, and for kind hospitality during my stays at these institutes. I would also like to thank Dr. J. J. Zijlstra (Netherlands Institute for Sea Research, 
Texel) for his interest and critical remarks, and Professor Dr. L. B. Holthuis (State Museum for Natural History, Leiden) for solving taxonomical questions.

\section{LITERATURE CITED}

Chamberlain, J. A. (1976). Flow patterns and drag coefficients of cephalopod shells. Palaeontology, 19 (3), 539-563.

Childress, J. J. and Nygaard, M. (1974). Chemical composition and buoyance of midwater crustaceans as a function of depth of occurrence of southern California. Mar. Biol., $27,225-238$.

Culkin, F. and Morris, K. J. (1969). The fatty acids of some marine crustaceans. Deep Sea Res., 16, 109-116.

Denton, E. J. (1961). The buoyance of fish and cephalopods. Prog. Biophys., 11, 177-235.

Denton, E. and J. Gilpin-Brown, J. B. (1961). The buoyance of the cuttlefish, Sepia officinalis. J. mar. biol. Ass. U. K, 41, 319-342.

Glasstone, S. and Lewis, D. (1966). Elements of Physical Chemistry, Macmillan \& Co, London.

Gross, F. and Raymond, J. E. G. (1942). The specific gravity of Calanus finmarchicus. Proc. R. Soc. Edinb., B 61, 288-296.

Gross, F. and Zeuthen, E. (1948). The buoyance of plankton diatoms: a problem of cell physiology. Proc. R. Soc., B 135, 382-389.
Lowndes, A. G. (1938). The density of some living aquatic organisms. Proc. Linn. Soc. Lond., 150, 62-73.

Lowndes, A. G. (1942). The displacement method of weighing living aquatic animals. J. mar. biol. Ass. U. K., 25, 555-574.

Lowndes, A. G. (1943). Some applications of the displacement method for weighing living aquatic organisms. Proc. zool. Soc, Lond., 13 A, 28-43.

Morris, R. W. (1971). Comparison of the fat composition of oceanic copepods from different depths. Comp. Biochem. Physiol., 40 B $275-281$.

Nicol, J. A. C. (1960). The Biology of Marine Animals, Wiley, London.

Pedley, T. J. (1977). Scale Effects in Locomotion, Academic Press, London.

Riley, J. P. and Skirrow, G. (1975). Chemical Oceanography, 2nd ed., Vol. IV. Academic Press, London.

Scott, W. J. and Chamberlain, J. A. (1976). Equations of motion for post mortum sinking of cephalopod shells and the sinking of Nautilus. Palaeobiology, 2, 8-18.

Spaargaren, D. H. (1972). Osmoregulation in the prawns Palaemon serratus and Lysmata seticaudata from the bay of Naples. Neth. J. Sea Res., 5, 416-436.

Spaargaren, D. H. (1980). Hydrodynamic properties of benthic marine crustacea: II. Energy requirements for maintaining vertical position. Mar. Ecol. Prog. Ser., 2, in press.

$\mathrm{Wu}, \mathrm{T}$. J. (1977). Introduction to the scaling of aquatic animal locomotion. In: Pedley, T. J. (ed.) Scale effects in locomotion. Academic Press, London, pp. 203-233.

This paper was submitted to the editor; it was accepted for printing on November 14, 1979. 